\title{
Proposed Use of Monte Carlo Simulated Images to Evaluate the Accuracy of Measurements on X-Ray Computed Tomography
}

\author{
Tomasz Kowaluk, Maciej Maciak, Adam Woźniak, Piotr Tulik, Natalia Golnik \\ Institute of Metrology and Biomedical Engineering, Faculty of Mechatronics, Warsaw University of Technology, \\ św. A. Boboli 8 st., 02-525 Warsaw, Poland, t.kowaluk@mchtr.pw.edu.pl
}

\begin{abstract}
$\mathrm{X}$-ray computed tomography $(\mathrm{CT})$ is increasingly recognized as a promising measuring technique for dimensional metrology. Various methods are being developed to improve measurement accuracy. Tests of new methods for such applications include accuracy evaluation with the use of calibrated workpieces; however, the internal algorithms of image acquisition and data processing might influence the experimental error, and then also the comparison of methods at different CTs. The accuracy of the results of tomographic measurements is influenced by many factors, one of which is the setting of the threshold value. The article presents the results of an attempt to use Monte Carlo simulated images to estimate deviations to determine threshold values to improve measurement accuracy and additionally, to estimate the impact of data processing. The differences of the results obtained from the simulated images were up to $4 \%$ larger than those from tomographic images. It was caused by degradation of the image contrast by scattered radiation.
\end{abstract}

Keywords: Monte Carlo Methods, X-ray computed tomography, accuracy of measurements, threshold values

\section{INTRODUCTION}

X-ray computed tomography as a medical tool has its origins in the 1970s. In recent years it is also increasingly recognized as a promising measuring technique for dimensional metrology [1]-[4]. This three-dimensional measurement technique enables the inspection of parts' internal and external features in a non-destructive way, and the creation of a 3D model for the entire volume of a scanned object. It also has the ability to generate geometric data for the characterization of material structures and detection of manufacturing imperfections and faults.

The new class of coordinate measuring machines allows complete measurements of complex objects with internal structures of even several hundred dimensions in a relatively short time of less than 30 minutes. The accuracy ranges from a few microns for standard applications, to fractions of a micron for precision measurements. The use of these devices leads to numerous applications and one of them is measuring voids/porosity morphology, and distribution [5]-[8].

Tomographic measurement accuracy is affected by a number of factors such as instability of the radiation source and detector, scattering of the radiation beam in the material, scatter and beam hardening, the impact of external factors, reconstruction algorithms errors, magnification setting or threshold value setting. One of the main factors is threshold value setting. Several threshold correction methods can be found in the literature [9]-[10]. A new method for such applications has been recently developed in our Department [11]. The method involves executing additional calibration measurement and determining certain corrections PS for airmaterial threshold values on the greyscale of the volumetric tomographic files. The PS deviation is the percentage difference between the surface area of the measured object (reference sphere - the surface area of the circle calculated as the sum of pixels on the X-ray images) registered on the detector and the reference value (the surface area of the circle with the diameter of the reference ball).

The proposed method requires a whole set of suitable reference workpieces to determine the PS value. For this reason, it was decided to try to determine the PS deviation value using Monte Carlo simulated images. A generally accepted method for accuracy evaluation is the use of reference workpieces. For this work, it was decided to use a set of calibration spheres. Then, X-ray simulation with FLUKA Monte Carlo code [12]-[13] was performed, in order to get a machine-independent image. It is defined as distribution of X-ray fluence on the detector surface representing directly the attenuation of X-ray radiation through the sample. Tests of determination of PS correction values were performed using both - the real images and the simulated ones. 


\section{MEASUREMENTS}

The measurements were performed using Carl-Zeiss METROTOM 800 cone beam CT. The photon source was Hamamatsu micro-focus X-ray tube (type L8032) with a Tungsten anode operated at a high voltage of $130 \mathrm{kV}$ [14]. The source is $5 \mu \mathrm{m}$ in diameter. A $100 \mu \mathrm{m} \mathrm{Be} \mathrm{window}$ separates the vacuum chamber and the surrounding air. A $500 \mu \mathrm{m}$ aluminum attenuator is used to reduce the primary flux of low energy photons. The emission cone of the X-ray source is $39^{\circ}$. Behind the source and the attenuator X-rays are collimated so that the primary rays are directed only towards the active part of the detector. The opening angle of the beam in the trans-axial plane, or fan-angle, is $32^{\circ}$, and the opening angle along the axis of the scanner, or cone-angle, is $8^{\circ}$. The tomograph is equipped with Varian amorphous silicon digital X-ray PaxScan detector type $2520 \mathrm{~V}$ incorporating sensor array with a cesium iodine scintillator. The total pixel matrix is $1536 \times 1920$ with pixel pitch equal to $127 \times 127 \mu \mathrm{m}^{2}$.

A set of 7 reference spheres (ball bearings), with diameters from 3 to $50 \mathrm{~mm}$, made of $\mathrm{Si}_{3} \mathrm{~N}_{4}$ served as the test workpieces. The reference diameter values were assumed on the basis of technical documentation. The measurements of the spheres' diameter in the images were performed in two series. The dependence of the PS deviation on the distance between the workpiece and radiation source was studied in the first series for the $13 \mathrm{~mm}$ sphere, while the second series was devoted to the determination of the influence of the object's size, when the object is in optimum position versus the radiation source.

Measurements were made using the METROTOM OS software. In case of actual measurements, the setting of the element has a significant effect on the value of the PS deviation. Offset of the reference workpiece from the axis of rotation will change the value of the cross-section area of the sphere depending on the angular setting. For this reason, each measurement was made from 1500 projections, recorded every 50 X-ray images, and for each image the PS value was determined according to [11]. The obtained values of the deviations were determined as the average value of PS for comparison with the results from the simulation. In the case of simulation, it was assumed that the measuring element was centered and that the mean value was not determined.

\section{Monte CARlo Simulations}

In order to describe the complete chain of $\mathrm{CT}$, the full models include simulating all necessary properties of X-ray sources, the interaction of photons with material with special attention to scattered radiation, detection process, and the complete geometrical CT setup. Such simulations can provide accuracy and are applicable for different purposes, such as the qualification of systems, the optimization of system parameters, feasibility analysis, model-based data interpretation, and others [15]-[19].

In this work, the interest was focused on the degradation of the image contrast by scattered radiation, for the test objects. It can be estimated, basing on physical principles of radiation interaction with matter, that expected scatter-to-primary ratio (SPR) is of about $5 \%$ for our test object. Details of the image were not important, so the simplified geometrical model of the radiation source could be used. Much more challenging was simulating the detector as a large amount of photon interactions as it had to be simulated so as to obtain a noisefree Monte Carlo estimate.

The work makes use of the FLUKA computing environment based on the MCM (Monte Carlo Method). FLUKA [12]-[13] is a universal tool aimed at estimating the transport and interaction of particles with matter. Its wide application range includes calculating the shields for electron and proton accelerators, designing their shields, calorimetry, dosimetry, designing detectors, cosmic radiation, neutrino physics, radiotherapy, etc.

Geometrical model of the tomograph was made with the use of Flair environment [20], a graphical interface with a geometry editor. Geometrical data concerning the X-ray tube and the body of a tomograph were taken from the technical documentation of the device [14]. A number of constructional simplifications (e.g., no tomograph enclosure, positioning system, clamping device) were used due to the significant correlation between the computational speed and the complexity of the geometrical model, and, at the same time, negligible influence of additional details of a geometrical model on the final results.

Geometrical model of the device consists of a simplified model of an X-ray tube, the inspected object and a detector matrix (Fig.1.). X-ray tube has real dimensions of the casing, elements of a collimator and a beryllium window. The spot focal point of the X-ray tube adopted in the model was located in the middle of the plane of a beryllium window. The detector matrix was represented as a virtual object suspended in space and having a grid of detectors corresponding to the actual matrix of the detectors. The matrix is $1920 \times 1536$ pixels with a single pixel of $127 \times 127 \mu \mathrm{m}^{2}$. The assumed thickness of the matrix is $127 \mu \mathrm{m}$, thus the created voxels (bins) are cubes with a side of $127 \mu \mathrm{m}$. The nominal distance between the focal point of the X-ray tube and the detector matrix (in fact it is the distance between beryllium window and a matrix) is $800 \mathrm{~mm}$, however, in practice, this is a variable value determined while calibrating the device. For the model, the mean value for this parameter was $789.587 \mathrm{~mm}$.

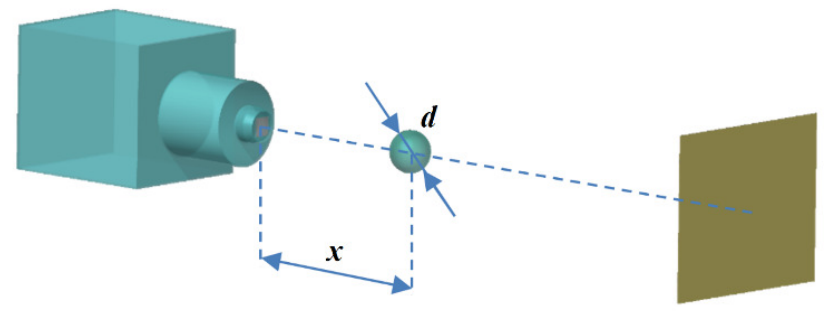

Fig.1. Visualization of the measuring system: $\mathrm{X}$-ray tube - object- detector matrix.

The source of radiation was defined as a mono-energetic point source of photons with the energy of $130 \mathrm{keV}$ propagating the photons isotropically. The materials assigned to particular elements of the model were selected from the Flair base, it was only necessary to define silicon nitride. 
Variable parameters in the numerical model were the diameters of the measured spheres made of silicon nitride $\mathrm{Si}_{3} \mathrm{~N}_{4}-d$ and the distance of the centers of the measured elements from the source of X-rays $-x$.

The value counted in each of the given bins was the number of all quanta of electromagnetic radiation reaching the detector, called particle/photon fluence.

As it can be seen, the model of the system was significantly simplified in terms of geometry and source definition. On the other hand, it was a serious challenge to define a matrix including almost 3 million bins, where the radiation quanta were counted. Simulation process required to perform such number of courses and simulation cycles which resulted in statistical error of less than $10 \%$. Statistical error, called relative error in Monte Carlo calculations, is a fractional standard deviation, defined as standard deviation divided by the mean and presented as percentage value. This is the value of error for which the obtained results may be interpreted as reflecting the actual probability of their occurrence. In order to obtain the results in reasonable time, it was necessary to use a computing cluster and parallel calculations.

\section{RESULTS}

As a result of the simulation, distribution of the total fluence of the X-radiation quanta on the plane of the matrix is achieved, analogical to the X-ray image obtained during tomographic acquisition. Only one simulated image is needed because there is no need to verify the effect of non-centricity. Verification of the model consists in comparing the results obtained from the simulated images with those obtained for CT scans (comparing the percentage value of the deviation of the cross-sectional area in terms of the default value - PS deviation). The deviation values of PS from both methods are determined according to [11]. Example image of a $50 \mathrm{~mm}$ sphere, obtained by MCM simulation and actual measurement, is shown in Fig.2.
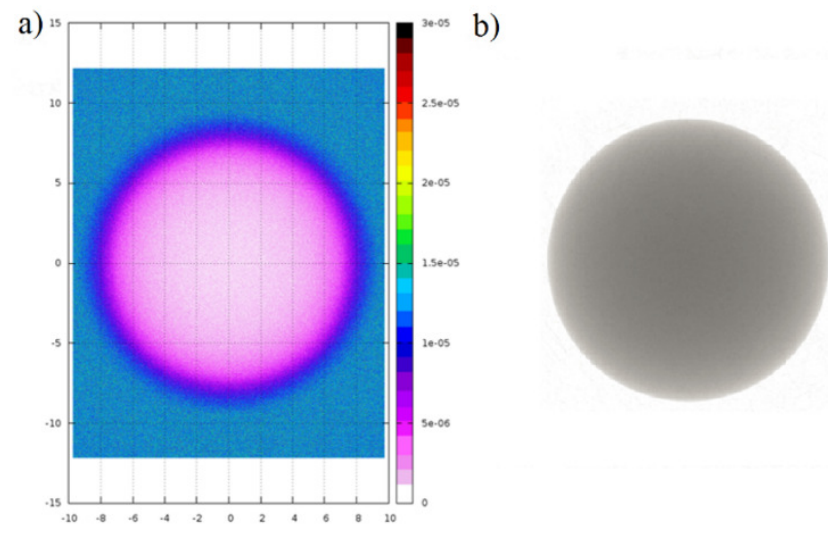

Fig.2. Exemplary illustration of the X-ray images: a) simulation, b) actual measurement.

In this work two types of X-ray images of test workpieces were used. The first one was obtained directly from X-ray computed tomography (CT), Fig.2.b), and the second one as a result of numerical simulation based on MCM, Fig.2.a). It is important to note that $\mathrm{CT}$ image acquisition is preceded by internal data processing dependent on the software coupled with the CT machine. End-user has no information about the level of processing of the image and algorithms applied during the process. In the case of MCM image the user gets an image that is a direct representation of $\mathrm{X}$-ray radiation attenuation level projected on the surface of the scintillator array. The image is not processed, each voxel simply has a numerical information about the total fluence of X-ray radiation in that point.

In measurements from CT scans, the values of the differences between the results and reference values did not exceed $1 \%$. The errors of the results of the obtained images, simulated on the basis of $10^{10}$ of the photon histories, are within $\pm 4 \%$.

A comparison of the obtained mean values (for ten measurements) of the deviation of PS as a function of the distance from the radiation source is shown in Fig.3.

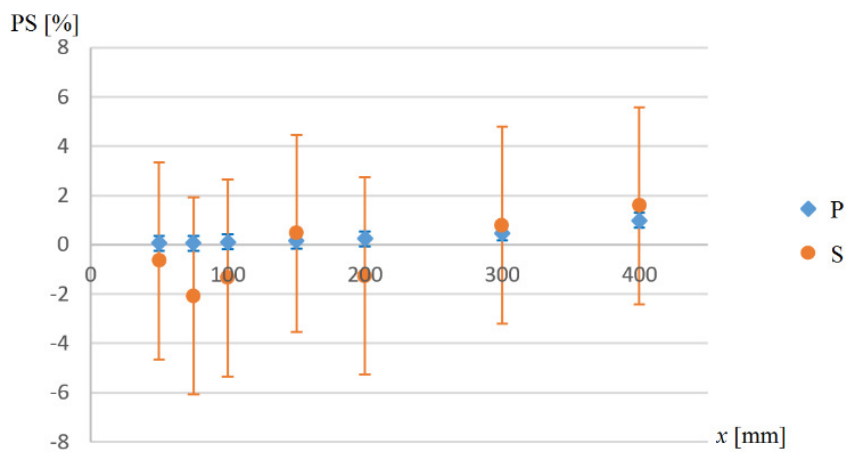

Fig.3. A graph illustrating the value of the deviations of the PS as a function of the distance of the object from the radiation source $x$. $\mathrm{S}$ - simulations, $\mathrm{P}$ - results of experimental measurements.

In measurements from CT scans, the achieved values of the cross-sectional areas were higher than reference values in all cases. In the results from simulation, both - positive and negative deviations were observed. Together with the increase in the distance from the source of radiation, the value of error also increases. Considering the obtained values of the simulation errors and the results obtained from the actual measurements considering the range value, it should be noted that $100 \%$ of the actual results fall within the range of the values obtained for the simulation. Because the results obtained from the simulation are characterized by significant error values ( $8 \%$ range) compared to actual measurements $(0.06 \%$ range), there is no basis for using them to determine the actual deviation of PS values.

In order to enable estimation of the value of a PS deviation with the values of the distances of the measuring element from the source of radiation other than tested, the results of the tests were matched with the mean values obtained from measurements.

When analyzing the results, one can assume that the percentage values of error may be determined at any distance by using the quadratic function defined as follows (1):

$$
P S=0.0000058 x^{2}+0.0296745
$$

where: 
$x$ - the distance of the measured object from the source of radiation in $\mathrm{mm}$.

Adjusting the function is shown in Fig.4. (correlation coefficient $0.9947 ; \mathrm{R}^{2}-98.95 \%$ ).

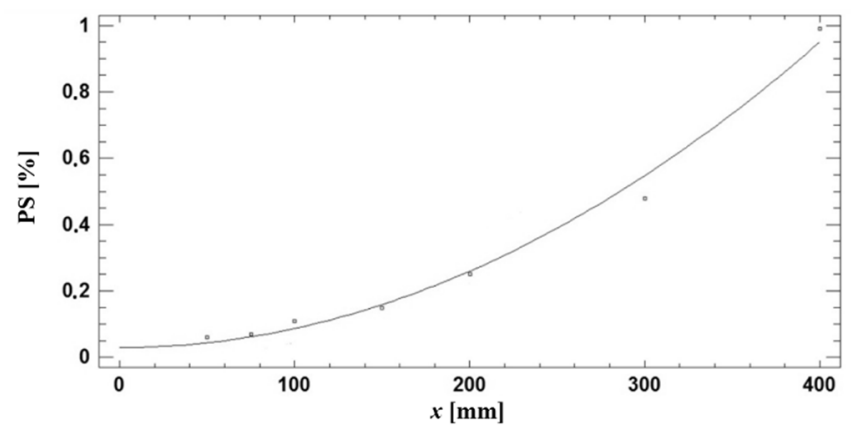

Fig.4. A chart of the values of the PS errors with a regression model (1) as a function of distance $x$ of the measured object from X-ray tube.

As in the majority of measurements, the measuring element is placed as close to the source of radiation as possible in order to get the best possible resolution of the measurements, it was decided to check the variability of the obtained values of errors as a function of the diameter $d$ of the measured detail. The distance from the tube was set in such a way that the entire element was seen on a detector at maximal magnification. Summary of the obtained results of the mean values from real measurements, as well as simulations, are presented on graph (Fig.5.).

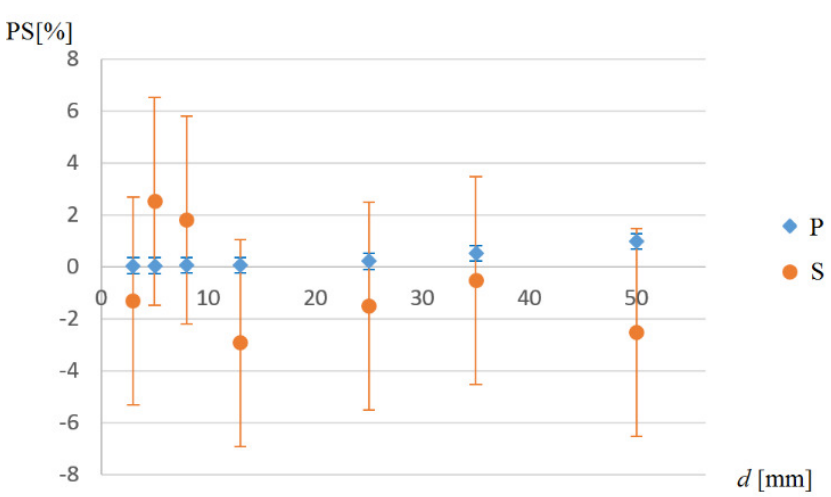

Fig.5. A graph illustrating the value of the deviations of the PS as a function of the diameter of the measured object $d$. S - simulations, $\mathrm{P}$ - results of experimental measurements.

Dependencies analogical to those from Fig.3. were observed in measurements, what obviously is related to the fact that the optimal distance from the source for a given test object is correlated with its size. The value of error, depending on the size of the measured element, can be estimated by the quadratic function (correlation coefficient $0.9975 ; \mathrm{R}^{2}$ equal to $99.51 \%$ ). Adjusting the function is shown in Fig.6. The determined function is described as follows:

$$
P S=0.00039 d^{2}+0.01947
$$

where:

$d$ - the diameter of the measured object.

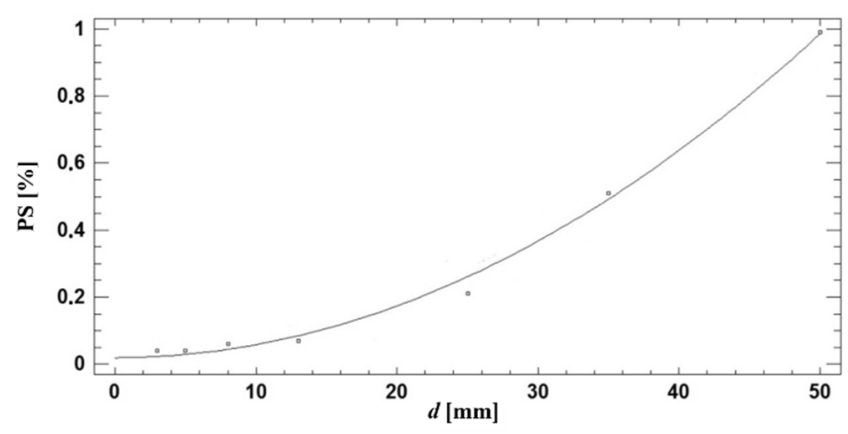

Fig.6. A chart of the PS error values with the regression model (2) as a function of the diameter of the measured object.

\section{CONCLUSIONS}

The work has checked whether it is possible to use Monte Carlo simulated images to determine PS deviation values to improve the accuracy of measurements on X-ray computed tomography. The essence of this validation was to use artificially obtained raw images of the test workpiece, resulting from computer simulation. Percentage values of the deviation of the cross-sectional area in terms of the default value were obtained for real CT scans as well as for simulated images.

The use of the Monte Carlo methods is possible, however, test results are not satisfactory. The results obtained from actual measurements have lower percentage error values. The observed errors of the measured values were up to $3 \%$ for simulated images and up to $1 \%$ for the images of the CT machine. Much smaller error for CT images is probably related to internal mathematical algorithms applied for raw image processing. Unfortunately, there is no way to obtain raw data from CT machines and compare them in that form with simple simulated images. Further improvements in calculation code are planned to model more precise conditions of image generation by the simulation approach.

Actual measurements' dependence on the distance of the measured element from the source of X-ray radiation and dependence on the size of the measured element were defined in this paper. In both cases quadratic functions to estimate percentage PS deviation were given.

\section{ACKNOWLEDGMENT}

The research has been co-funded by the Ministry of Science and Higher Education in Poland, decision No. 5339/E365/SPUB/2016/1 and 5339/E-365/SPUB/2017/1.

\section{REFERENCES}

[1] Maire, E., Withers, P.J. (2014). Quantitative X-ray tomography. International Materials Reviews, 59 (1), $1-43$.

[2] Kruth, J.P., Bartscher, M., Carmignato, S., Schmitt, R., De Chiffre, L., Weckenmann, A. (2011). Computed tomography for dimensional metrology. CIRP AnnalsManufacturing Technology, 60 (2), 821-842. 
[3] De Chiffre, L., Carmignato, S., Kruth, J.P., Schmitt, R., Weckenmann, A. (2014). Industrial applications of computed tomography. CIRP Annals-Manufacturing Technology, 63, 655-677.

[4] Villarraga-Gómez, H. (2016). X-ray computed tomography for dimensional measurements. In Digital Imaging 2016: ASNT Topical Conference, Mashantucket, CT, USA. American Society for Nondestructive Testing, 44-57.

[5] Villarraga-Gómez, H. (2016). Seeing is believing: Xray computed tomography for quality control. Quality Magazine, 55 (6), 20-23.

[6] Villarraga-Gómez, H., Lee, C., Corbett, T., Tarbutton, J.A., Smith, S.T. (2015). Assessing additive manufacturing processes with X-ray CT metrology. In ASPE Spring Topical Meeting, Raleigh, NC, USA. Vol. 60, 116-120.

[7] Gapinski, B., Wieczorowski, M., Marciniak-Podsadna, L., Dybala, B., Ziolkowski, G. (2013). Comparison of different method of measurement geometry using CMM, optical scanner and computed tomography 3D. In 24th DAAAM International Symposium on Intelligent Manufacturing and Automation.

[8] Hermanek, P., Carmignato, S. (2016). Reference object for evaluating the accuracy of porosity measurements by X-ray computed tomography. Case Studies in Nondestructive Testing and Evaluation, 6, 122-127.

[9] Kiekens, K., Welkenhuyzen, F., Tan, Y., Bleys, P., Voet, A., Kruth, J.-P., Dewulf, W. (2011). A test object with parallel grooves for calibration and accuracy assessment of industrial computed tomography (CT) metrology. Measurement Science and Technology, 22 (11), 115502.

[10] Obrist, A., Flisch, A., Hofmann, J. (2004). Point cloud reconstruction with sub-pixel accuracy by sliceadaptive thresholding of X-ray computed tomography images. NDT \& E International, 37, 373-380.

[11] Kowaluk, T., Wozniak, A. (2017). A new threshold selection method for X-ray computed tomography for dimensional metrology. Precision Engineering, 50, 449-454.

[12] Battistoni, G., Muraro, S., Sala, P.R., Cerutti, F., Ferrari, A., Roesler, S., Fasso, A., Ranft, J. (2007). The FLUKA code: Description and benchmarking. In AIP
Conference Proceedings of the Hadronic Shower Simulation Workshop 2006, 6-8 September 2006. AIP, Vol. 896, 31-49.

[13] Böhlen, T.T., Cerutti, F., Chin, M.P.W., Fassò, A., Ferrari, A., Ortega, P.G., Mairani, A., Sala, P.R., Smirnov, G., Vlachoudis, V. (2014). The FLUKA Code: Developments and challenges for high energy and medical applications. Nuclear Data Sheets, 120, 211-214.

[14] Hamamatsu Photonics K.K. 130 kV Microfocus X-Ray Source, L9181-02. Instruction Manual. https://www.hamamatsu.com/resources/pdf/etd/L9181 -02_TXPR1015E.pdf.

[15] Berdondinia, A., Bettuzzia, M., Bianconia, D., Brancaccioa, R. (2007). Monte Carlo optimization of an industrial tomography system. Nuclear Instruments and Methods in Physics Research A, 580, 771-773.

[16] Sladek, J., Gaska, A. (2012). Evaluation of coordinate measurement uncertainty with use of virtual machine model based on Monte Carlo method. In Measurement, 45 (6), 1564-1575.

[17] Hiller, J., Reindl, L. (2012). A computer simulation platform for the estimation of measurement uncertainties in dimensional X-ray computed tomography. Measurement, 45 (8), 2166-2182.

[18] Miceli, A., Thierrya, R., Flischa, A., Sennhausera, U., Casalib, F., Simonc, M. (2007). Monte Carlo simulations of a high-resolution X-ray CT system for industrial applications. Nuclear Instruments and Methods in Physics Research A, 583, 313-323.

[19] Chyba, B., Mantler, M., Reiter, M. (2008). Monte Carlo simulation of projections in computed tomography powder diffraction. Powder Diffraction, 23 (2), 150153.

[20] Vlachoudis, V. (2009). FLAIR: A powerful but user friendly graphical interface for FLUKA. In International Conference on Mathematics, Computational Methods \& Reactor Physics (M\&C 2009), Saratoga Springs, New York, 3-7 May 2009. American Nuclear Society.

Received May 08, 2018 Accepted November 03, 2018 